\title{
Exclusive breastfeeding for under-6-month-old children
}

\author{
Sara B. Fein*
}

In this issue of Jornal de Pediatria, Parizoto et al. ${ }^{1}$ provide the first description and analysis of trends in rates of exclusive breastfeeding from the public health surveillance project "Breastfeeding and Municipalities" conducted in the city of Bauru, state of São Paulo between 1999 and 2006. They also analyze various factors that may be associated with exclusive breastfeeding.

Public health surveillance may be defined as ongoing and systematic collection and analysis of data about specific health behaviors, conditions, or other topics. Such surveillance is essential for improving health because it enables health agencies to determine the levels of health behavior in the population, whether these levels are increasing or not, and whether any improvement is experienced by all groups or only some. Surveillance of trends in small geographical areas has the advantage that community level variables and community level changes are likely to be the same for all mothers studied, and therefore the effects of community level interventions may be more easily identified if other areas are available for comparison. The particular surveillance system analyzed here has results reported for a number of different communities, which enables a comparison with those other communities. Analysis of the trends is a key component of effective surveillance, and analysis of associated factors is also useful for understanding which groups are in greater need of support or special services and what types of interventions are more likely to improve the public's behavior.

Exclusive breastfeeding is important to infant health, as indicated by the World Health Organization (WHO) in their recommendation that infants be exclusively breastfed for 6 months. ${ }^{2}$ Health protection from exclusive breastfeeding is not limited to developing countries; a large-scale meta- analysis of the effect of breastfeeding on infant health outcomes in developed countries found that exclusive breastfeeding was more protective than partial breastfeeding for acute otitis media, atopic dermatitis, and hospitalization for respiratory illness. ${ }^{3}$

The results reported by Parizoto et al. ${ }^{1}$ are significant for several reasons. First, they show how this part of the state of São Paulo stands relative to other towns in the same state through their comparisons with published data from other areas of the state. These results also enable policy makers and program directors to see how this area of Brazil compares with overall national data on exclusive breastfeeding. Second, the results provide policy and program relevant details about infant ages when exclusive breastfeeding rates decrease and which groups of mothers have lower rates and thus need additional support. Third, the results add to the literature on the effects of a specific modifiable factor, pacifier use, which has consistently been found in observation studies to have a strong negative association with breastfeeding. The effects of pacifier use are of particular interest because in the USA, the American Academy of Pediatrics recommends pacifier use to reduce the risk of sudden infant death syndrome, ${ }^{4}$ although for breast-fed infants, they recommend that pacifiers not be offered until after 1 month of age.

Most of the results reported are consistent with other research throughout the world. The negative association between pacifier use and exclusive breastfeeding has been found in many countries, both developed ${ }^{5}$ and developing 6 and was acknowledged by the WHO in their 1991 Baby Friendly Hospital Initiative. ${ }^{7}$ As the authors carefully discuss, causation is still debated for this association. It is possible that sucking on a pacifier in itself inhibits breastfeeding, in which case discouraging mothers from using pacifiers will

* PhD. Consumer Studies Staff, Center for Food Safety and Applied Nutrition, Food and Drug Administration, College Park, Maryland, USA

No conflicts of interest declared concerning the publication of this editorial.

Suggested citation: Fein SB. Exclusive breastfeeding for under-6-month-old children. J Pediatr (Rio J). 2009;85(3):181-182. doi:10.2223/JPED.1905 
improve breastfeeding rates. Studies that have measured the effect of very early pacifier use on later breastfeeding outcomes 5 and those that have separated the effects of early breastfeeding problems from the effects of pacifier use $^{8}$ support this direction of causality. But it is also possible that mothers who have breastfeeding problems turn to pacifiers to soothe their infants, in which case the policy implication is that greater breastfeeding support is needed to keep the situations from developing that lead mothers to use pacifiers. And it is also possible that both of these directions of causality are operating.

Another result that was not statistically significant in this study showed a direction of association that is consistent with research from various other countries. Younger mothers have lower exclusive breastfeeding rates than older mothers, suggesting that extra effort and support are needed universally for young mothers.

The authors note that varying types of research have found that mothers who receive a greater number of breastfeeding services have higher rates of breastfeeding than mothers who receive fewer. This association has been found for breastfeeding supportive maternity care practices, ${ }^{5}$ as well as the settings cited in the article: number of breastfeeding support contacts in the WHO growth curve study in Brazil, ${ }^{9}$ number of prenatal sessions attended ${ }^{10}$ and community level pro-breastfeeding actions. ${ }^{11}$ The effectiveness of breastfeeding support from non-medical but trained peer counselors ${ }^{12}$ may be the result of this general phenomenon. Greater breastfeeding support is a modifiable factor, and as the success of peer counseling programs shows, does not have to be done by medical personnel for mothers with no complex breastfeeding problems.

This study has a number of strengths. The sample of the Breastfeeding and Municipalities survey is probably as representative as feasible of infants in each area, although it is unfortunate that the authors do not describe characteristics of mothers who attend the vaccination campaign. The questions to measure exclusive breastfeeding are strong; they ask for detailed feeding data that the researchers use to define exclusive breastfeeding rather than asking the mother to make the judgment herself. In addition, the interviewers were carefully trained.

The study also has several limitations. Most important, because it is cross-sectional, causality or even time order cannot be established. The authors cite longitudinal studies on some of the important issues, such as pacifier use and duration of exclusive breastfeeding, and this strategy provides logical interpretations for their results. Another limitation is that some important variables were not measured. The authors point out that the number of sessions of prenatal care has been found in other studies in Brazil to be positively related to breastfeeding duration, but this variable was not reported and apparently not measured in the survey. Similarly, maternity care practices related to breastfeeding, availability of professional breastfeeding support, and strength of social support were not measured. Because important variables were not included in the analysis, the conclusion that only pacifier use was significantly associated with exclusive breastfeeding needs to be considered in the context of the variables analyzed.

In addition, as the authors speculate on reasons for the increase in exclusive breastfeeding over the years of the three measurements, they discuss the likely effect of the community breastfeeding support campaign that had occurred since 2000 . They note that the campaign focused on health professionals working in public primary health care and maternity hospitals. However, the two measures they display regarding place of delivery and type of child medical care, which might be expected to indicate effectiveness of the campaign, were not significantly related to exclusive breastfeeding in the first 6 months.

\section{References}

1. Parizoto GM, Parada CM, Venâncio SI, Carvalhaes MA. Trends and patterns of exclusive breastfeeding for under-6-month-old children. J Pediatr (Rio J). 2009;85:201-208.

2. World Health Organization. Global Strategy on Infant and Young Child Feeding. Geneva: WHO; 2002.

3. Ip S, Chung M, Raman G, Chew P, Magula N, DeVine D, et al. Breastfeeding and Maternal and Infant Health Outcomes in Developed Countries. Evidence Report/ Technology Assessment No. 153 (Prepared by Tufts-New England Medical Center Evidencebased Practice Center, under Contract No. 290-02-0022). AHRQ Publication No. 07-E007. Rockville, MD: Agency for Healthcare Research and Quality; 2007.

4. American Academy of Pediatrics Task Force on Sudden Infant Death Syndrome. The changing concept of sudden infant death syndrome: diagnostic coding shifts, controversies regarding the sleeping environment, and new variables to consider in reducing risk. Pediatrics. 2005;116:1245-55.

5. DiGirolamo AM, Grummer-Strawn LM, Fein SB. Effect of maternitycare practices on breastfeeding. Pediatrics. 2008;122 Suppl 2: S43-9.

6. Koosha A, Hashemifesharaki R, Mousavinasab N. Breastfeeding patterns and factors determining exclusive breastfeeding. Singapore Med J. 2008;49:1002-6.

7. United Nations Children's Fund - UNICEF. The global criteria for the WHO/UNICEF Baby-Friendly Hospital Initiative. New York, NY: UNICEF; 1992.

8. Kronborg $\mathrm{H}$, Vaeth $\mathrm{M}$. How are effective breastfeeding technique and pacifier use related to breastfeeding problems and breastfeeding duration? Birth. 2009;36:34-42.

9. Albernaz E, Araújo CL, Tomasi E, Mintem G, Giugliani E, Matijasevich $A$, et al. Influence of breastfeeding support on tendencies of breastfeeding rates in the city of Pelotas (RS), Brazil, from 1982 to 2004. J Pediatr (Rio J). 2008;84:560-4.

10. Santo LC, de Oliveira LD, Giugliani ER. Factors associated with low incidence of exclusive breastfeeding for the first 6 months. Birth. 2007;34:212-9.

11. Venâncio SI, Monteiro CA. Individual and contextual determinants of exclusive breastfeeding in São Paulo, Brazil: a multilevel analysis. Public Health Nutr. 2006;9:40-6.

12. Shealy KR, Li R, Benton-Davis S, Grummer-Strawn LM. The CDC Guide to Breastfeeding Interventions. Atlanta, GA: US Department of Health and Human Services, Centers for Disease Control and Prevention; 2005.

Correspondence:

Sara B. Fein

E-mail: sara.fein@fda.hhs.gov 